\title{
Effects of Long Waves on Wind-generated Waves
}

\author{
Gang Chen* and Stephen E. Belcher \\ Department of Meteorology, University of Reading, Reading, United Kingdom
}

(Manuscript received 28 April 1999, in final form 25 October 1999)

Journal of Physical Oceanography, Vol. 30, No. 9, pp. 2246-2256 (2000)

Corresponding author address:

Dr. Gang Chen

Laboratoire Interactions Océan-Atmosphère

CNRS/Université de la Méditerranée

Campus de Luminy-Case 903

13288 Marseille Cedex 9

France

E-mail: chen@pollux.irphe.univ-mrs.fr

${ }^{*}$ On leave of absence from Laboratoire Interactions Océan-Atmosphère, CNRS/Université de la Méditerranée, Marseille, France. 


\begin{abstract}
A model is developed to explain the observation made in several laboratory experiments that short wind-generated waves are suppressed by a train of long, mechanicallygenerated waves. A sheltering mechanism is responsible for generation of the short wind waves, by which wave growth is proportional to the local turbulent wind stress. Hence, if the turbulent wind stress near the surface is reduced by the long wave, then the short wind wave amplitude, and hence also the energy in the short waves at a given fetch, is lower than in the absence of long wave. A quantitative model of this process is formulated to examine the ratios of the growth rate and the total energy density of wind waves with and without a long wave, which is shown to agree reasonably well with the laboratory experiments. The model also explains why this suppression of wind waves by a very long swell is not observed in the ocean where the effects of swell on wind waves are extremely difficult to detect. In the model, the reduction in the turbulent wind stress by the long wave is largest for small values of $C_{L} / u_{*}$ (where $C_{L}$ is the phase speed of the long wave and $u_{*}$ is the friction velocity of the wind). When this ratio is larger than about 25 (which is typical of ocean swell), both the reduction of the turbulent wind stress by the long wave, and consequently the reduction in the total energy density of the wind waves, are very small, which explains why this phenomenon has not yet been observed on the ocean.
\end{abstract}




\section{Introduction}

Several laboratory studies (e.g., Mitsuyasu 1966; Phillips and Banner 1974; Donelan 1987) have observed that when a train of long, mechanically-generated, waves are made to propagate in the same direction as the wind is blowing, then the short-wavelength wind-generated waves are suppressed. As the slope of the mechanical wave is increased, there is a reduction in the spectral density, particularly in the energy containing range, and thus the total energy density of the wind-generated waves. For example, with a long wave of slope, $a_{L} k_{L}$, of approximately 0.05 , Mitsuyasu (1966) report a reduction in the total energy density of the wind waves to less than $60 \%$ of the value in the absence of mechanical waves, while for $a_{L} k_{L}=0.105$, Donelan (1987) found that the total energy density of the wind waves was reduced by a factor of about 2.5. What are the mechanisms responsible for this striking phenomenon? And what is the implication for the effects of swell on wind waves on the ocean?

In deep water, without currents, the evolution of the frequency spectrum of wind waves, $\Phi(\sigma, x, t)$, is governed by the energy balance equation (see, for example, Komen et al. 1994, p 47):

$$
\frac{\partial \Phi}{\partial t}+C_{g} \frac{\partial \Phi}{\partial x}=S_{w}+S_{n l}+S_{d s}
$$

where $\sigma$ is the radian wave frequency and $C_{g}$ is the group velocity. The three source terms, $S_{w}, S_{n l}$ and $S_{d s}$, represent, respectively, energy input from the wind, nonlinear energy transfer due to wave-wave interactions, and energy dissipation due to wave breaking. The aforementioned laboratory measurements were made of a steady state so that the spectral density at any fetch and frequency is determined entirely by the upwind evolution of these source functions (which themselves are fetch dependent), namely

$$
\Phi(\sigma, x)=\int_{0}^{x} C_{g}^{-1}\left(S_{w}+S_{n l}+S_{d s}\right) d x
$$

The observed suppression of wind waves could therefore be the result of changes caused 
by the mechanical wave to any of these source functions. And indeed, several mechanisms have been put forward. Firstly, Phillips and Banner (1974) attributed this phenomenon to enhanced wave breaking (i.e., to $S_{d s}$ ). According to this idea, the wind-driven drift current is modulated by the long wave, which then enhances the breaking of short wind waves at the crest of the mechanical wave. However, Wright (1976) has shown that the Phillips-Banner mechanism is too weak at higher wind speeds and does not have the correct variation with wind speed. Secondly, Masson (1993) has suggested that nonlinear wave-wave interactions (i.e., $S_{n l}$ ) might be responsible for the reduction in the growth of the wind waves. Masson (1993) showed no quantitative comparisons with data, but the calculations do show that the nonlinear energy transfer between a long wave and wind waves decreases rapidly with the ratio of the peak frequency of the wind waves to the long wave frequency, with no significant energy transfer for ratios greater than about 1.6. Since the frequency ratio is greater than this in all the aforementioned laboratory experiments, this explanation does not seem viable.

In this paper we suggest that the suppression of wind waves by a long wave is due to direct coupling of the long wave with the wind (i.e., $S_{w}$ ). We follow an idea suggested by Makin et al. (1995), and more recently developed by Makin and Kudryavtsev (1999), in their calculation of the drag of the sea surface. Hence, we suppose that the long wave absorbs momentum from the wind as a drag on the long wave. Conservation of momentum then shows that this drag leaves a smaller turbulent momentum flux in the wind to generate waves, which therefore develop more slowly. A complete description of a quantitative formulation of this model, which is similar to the idea described briefly by Belcher and Chen (1999), is described in Section 2. Full, and new, quantitative comparisons with data are described in Section 3. In Section 4, we discuss the implications of this process on the effects of swell on wind waves on the ocean. Finally, a further discussion of the assumptions and conclusions are given in Section 5. 


\section{The model}

The model for how the long wave affects the wind-induced growth of the short waves is developed in three stages. Firstly, the wind-induced growth rate of the long wave is determined using results from previous studies. Secondly, this growth rate of the long wave is used to determine the fraction of the stress, or equivalently the momentum flux, carried in the wind that is absorbed by the long wave. Since the total stress is constant with height, we thus also determine the fraction of turbulent stress remaining in the wind that is available to grow short wind waves. A schematic of the configuration with wind blowing over wind waves and long waves, and the vertical profiles of contributions to the total stress is shown in Fig. 1. Thirdly, and finally, this reduced turbulent stress is used in a formula for the fetch-limited growth of wind waves that has been determined empirically in previous laboratory studies.

\section{a. Wind-induced growth of the long wave}

Consider a train of long, monochromatic, mechanically-generated waves, i.e., the long wave, which, in the absence of the wind, has angular frequency $\sigma_{L}$, amplitude $a_{L}$, wavenumber $k_{L}$, and hence a slope $a_{L} k_{L}$. This long wave propagates in the direction of the wind and grows with fetch due to the action of the wind (as observed by Mitsuyasu and Honda 1982). The wind-induced growth rate of the long wave, $\gamma_{L}$, is defined by

$$
\frac{\partial \Phi_{L}(\sigma)}{\partial t}=\gamma_{L} \Phi_{L}(\sigma)
$$

where $\Phi_{L}(\sigma)$ is the spectral density of the long wave.

Recent weakly-nonlinear analyses of wind over waves (van Duin 1996; Belcher 1999) 
have determined that the growth rate of a monochromatic wave is

$$
\gamma_{L}=\frac{\rho_{a}}{\rho_{w}} \frac{\alpha_{p}}{1+k_{L}^{2} \overline{\eta_{L}^{2}} \alpha_{p}}\left(\frac{u_{*}}{C_{L}}\right)^{2} \sigma_{L}=\frac{\rho_{a}}{\rho_{w}} \beta\left(\frac{u_{*}}{C_{L}}\right)^{2} \sigma_{L}
$$

where $C_{L}$ is the phase speed of the long wave, $\overline{\eta_{L}^{2}}$ is the variance of the long wave, which is $\frac{1}{2} a_{L}^{2}$ at fetch $x=x_{0}, \rho_{a}$ and $\rho_{w}$ are air and water densities, respectively, $u_{*}$ is the friction velocity of the wind and $\alpha_{p}$ is the asymmetric pressure coefficient (for details see Belcher 1999). The denominator in (4), namely $1+k_{L} \overline{\eta_{L}^{2}} \alpha_{p}$, is a weakly-nonlinear correction to the wave growth rate, which arises because the long wave absorbs momentum from the wind, thereby leaving a reduced level of turbulent wind stress near the surface to generate it by the non-separated sheltering mechanism (Belcher 1999). Finally, the growth rate coefficient, $\beta$, as defined in (4) varies with the ratio $C_{L} / u_{*}$ as shown in Fig. 2, which compares modeled values with data. The relationship between $\alpha_{p}$ and $\beta$ is immediately seen from (4).

\section{b. Partition between wave-induced stress and turbulent wind stress}

In the presence of surface waves, the wind velocity can be decomposed into three parts, $u=\bar{u}+\tilde{u}+u^{\prime}$, namely a time-averaged and spatially-averaged mean wind, $\bar{u}$, a waveinduced mean wind, $\tilde{u}$, and a turbulent wind fluctuation, $u^{\prime}$. Then the total stress in the wind, averaged over a wavelength of the waves, $\tau_{\text {tot }}$, is the sum of a turbulent stress, $\tau_{t}=-\rho_{a}\left\langle\overline{u^{\prime} w^{\prime}}\right\rangle$, and a wave-induced stress, $\tau_{L}=-\rho_{a}\langle\tilde{u} \tilde{w}\rangle$, where \langle\rangle refers to averaging over a wavelength of long waves. Hence the turbulent stress arises from vertical transport of horizontal momentum by the turbulent eddies and the wave-induced stress arises from vertical transport of horizontal momentum by the wave-induced flow.

When the wind and waves are both stationary in time and develop only very slowly with fetch (i.e., any changes with fetch are over many wavelengths of the waves), then the mean momentum equation shows that the total stress is constant with height (Townsend 
1972), so that

$$
\tau_{\text {tot }}=\tau_{t}+\tau_{L}=\text { constant }=\rho_{a} u_{*}^{2}
$$

Now, the generation of waves by wind is determined by the turbulent wind stress at the water surface. To calculate the turbulent wind stress, $\tau_{t}$, in the presence of the long wave, the wave-induced stress associated with the long wave, $\tau_{L}$, is first determined. The wave-induced stress at the surface is equal to the rate of change of the momentum in the wave, hence for the long wave

$$
\tau_{L}=\int_{0}^{\infty} \frac{\partial M_{L}(\sigma)}{\partial t} d \sigma
$$

where $M_{L}(\sigma)$ is the momentum density of the long wave, which is defined by (see, for example, Phillips 1977, p 40)

$$
M_{L}(\sigma)=\rho_{w} \sigma_{L} \Phi_{L}(\sigma)
$$

Substituting (7) into (6) and using the wind-induced growth rate, $\gamma_{L}$ [defined in (3)], yields the following expression for $\tau_{L}$ :

$$
\tau_{L}=\rho_{w} \int_{0}^{\infty} \gamma_{L} \sigma_{L} \Phi_{L}(\sigma) d \sigma
$$

Equation (4) for the wind-induced growth rate of the long wave then shows that the stress induced by the long wave becomes

$$
\tau_{L}=\tau_{t o t} \frac{\alpha_{p}}{1+k_{L}^{2} \overline{\eta_{L}^{2}} \alpha_{p}} k_{L}^{2} \overline{\eta_{L}^{2}}
$$

Under a steady wind, the amplitude of the long wave develops with fetch (Mitsuyasu and Honda 1982), and from the results described above the relative dependence can be written

$$
\epsilon_{L}=2 \overline{\eta_{L}^{2}}(x) / a_{L}^{2}\left(x_{0}\right)=\mathrm{f}\left(\epsilon_{L} ; a_{L} k_{L}, \alpha_{p}, \sigma_{*}, X_{*}-X_{*_{0}}\right)
$$

where the fetch dependence for deep water waves is written in dimensionless form as follows

$$
\mathrm{f}=\exp \left[\frac{2 \gamma_{L}}{C_{L}}\left(x-x_{0}\right)\right]=\exp \left[2 \frac{\rho_{a}}{\rho_{w}} \frac{\alpha_{p}}{1+\frac{1}{2}\left(a_{L} k_{L}\right)^{2} \alpha_{p} \epsilon_{L}} \sigma_{*}^{4}\left(X_{*}-X_{*_{0}}\right)\right]
$$


Here $\sigma_{*}=\sigma_{L} u_{*} / g$ is the dimensionless frequency of the long wave, $X_{*}=g x / u_{*}^{2}$ is the dimensionless fetch, $g$ is the acceleration due to gravity, $x$ is the fetch and $x_{0}$ may be considered to be the minimum fetch at which the wind begins to influence the long wave (Mitsuyasu and Honda 1982). This relative variance, which is greater than unity for a positive value of $\alpha_{p}$, is determined iteratively for a given set of dimensionless parameters, $a_{L} k_{L}, \alpha_{p}, \sigma_{*}$ and $X_{*}-X_{*_{0}}$.

Therefore, on using (9) and (10), the ratio of the stress induced by the long wave to the total stress is given by

$$
\frac{\tau_{L}}{\tau_{\text {tot }}}=\frac{\frac{1}{2}\left(a_{L} k_{L}\right)^{2} \alpha_{p} \epsilon_{L}}{1+\frac{1}{2}\left(a_{L} k_{L}\right)^{2} \alpha_{p} \epsilon_{L}},
$$

which, for a long wave of small slope, and for short fetch is approximately $\frac{1}{2}\left(a_{L} k_{L}\right)^{2} \alpha_{p}$.

The turbulent wind stress at the water surface, $\tau_{t}$, which is available to generate wind waves, is obtained from (5) and can be evaluated using (12) for the stress induced by the long wave to give

$$
\frac{\tau_{t}}{\tau_{\text {tot }}}=1-\frac{\tau_{L}}{\tau_{\text {tot }}}=\frac{1}{1+\frac{1}{2}\left(a_{L} k_{L}\right)^{2} \alpha_{p} \epsilon_{L}} .
$$

Note that for a positive $\alpha_{p}$, the ratio $\tau_{t} / \tau_{\text {tot }}$ is less that unity but bounded below by zero, as it should be. Equation (13) shows therefore how the long wave reduces the turbulent wind stress at the sea surface. Now, according to the non-separated sheltering mechanism, the wind generates waves in proportion to the turbulent stress at surface (Belcher and Hunt 1993, Belcher 1999) and hence the wind waves will be reduced in the presence of the long wave. In addition this equation shows that there are four dimensionless parameters that determine the magnitude of the effect: the initial slope of the long wave, $a_{L} k_{L}$; the asymmetric pressure coefficient, $\alpha_{p}$, which is related to the growth rate coefficient, $\beta$, as defined in (4); the dimensionless long wave frequency, $\sigma_{*}$; and the effective dimensionless fetch, $X_{*}-X_{*_{0}}$. The turbulent wind stress is reduced as these parameters increase, with a particular sensitivity to increasing the slope of the long wave as might be expected. Equation (13) will be used in the subsequent analysis of how 
the long waves affect development of wind waves.

\section{c. Effect of the long wave on development of wind waves}

Before investigating the effects of the long wave on wind-induced growth of the short waves, we recall some empirical relations for the development of pure wind waves (with no long wave) under steady wind. Since we are to compare the model with laboratory data, we use the fetch relations established by Mitsuyasu and Rikiishi (1978) in laboratory measurements of wind-wave development, which yield

$$
\frac{U_{*} \sigma_{p}}{g}=7.48\left(\frac{g x}{U_{*}^{2}}\right)^{-0.357},
$$

for development with fetch of the spectral peak frequency, $\sigma_{p}$, and

$$
\frac{g E}{\rho_{w} U_{*}^{4}}=4.49 \times 10^{-5}\left(\frac{g x}{U_{*}^{2}}\right)^{1.282},
$$

for development with fetch of the energy density of the wind waves, $E=\rho_{w} g \overline{\eta^{2}}$.

Now we turn to the development of the wind-waves in the presence of the long wave. And here we make a crucial assumption, namely that the only effect of the long wave on the development of the wind waves is to change the turbulent wind stress, $\tau_{t}$, at the water surface. Hence the fetch laws quoted above, and which were obtained in the absence of a long wave, are assumed to hold both with and without a long wave. Therefore, the friction velocity, $U_{*}$, in (14) and (15) is assigned the value of the local turbulent wind stress, so that $U_{*}=\left(\tau_{\text {tot }} / \rho_{a}\right)^{1 / 2}=u_{*}$ with no long wave and $U_{*}=\left(\tau_{t} / \rho_{a}\right)^{1 / 2}$ with the long wave.

Thence, using (14) and (15), the development with fetch of the peak frequency and total wind-wave energy are obtained with and without the long wave. The development of these quantities with and without the long wave can be compared simply on recognition that $\tau_{\text {tot }}$ has the same value with and without the long wave. This is true because, by 
definition, $\tau_{\text {tot }}$ is the total drag that the wave surface exerts on the wind, and hence in a stationary flow this drag balances the pressure gradient that forces the wind (the synoptic pressure gradient over the ocean or the pressure gradient down the tunnel in the laboratory). The pressure gradients must be the same for sensible comparisons with and without the long waves (which in the laboratory implies the same fan speed) and hence so too is $\tau_{\text {tot }}$.

Remy and Giovanangeli (1999) provide experimental evidence in support of this conclusion. An oblique long wave, with slope $a_{L} k_{L}=0$ (pure wind waves), $0.09,0.14$ or 0.2 was generated with a paddle that propagated at 35 degrees to the mean wind direction. The ratio $C_{L} / u_{*}$ ranges from $3.1\left(a_{L} k_{L}=0.2\right)$ to $6.2\left(a_{L} k_{L}=0.09\right)$. In each case, the wavelength of the long wave was six times the wavelength of the short wind waves at the spectral peak. They measured the total stress using both the inertial-dissipation method and eddy-correlation method. In Fig. 3, we plot the values of the total stress, $\tau_{\text {tot }} / \rho_{a}$, measured by Remy and Giovanangeli (1999) against slope of the long wave. To the accuracy of the measurements the total stress is clearly unaffected by the long wave, as argued above.

Hence, from (14), if $\sigma_{0_{p}}$ is the frequency of the spectral peak of wind waves in the absence of long wave, then

$$
\frac{\sigma_{p}}{\sigma_{0_{p}}}=\left(\frac{\tau_{t}}{\tau_{t o t}}\right)^{-0.143},
$$

which implies that the spectral peak frequency has higher values when a long wave is added to wind waves, in qualitative agreement with laboratory observations (Donelan 1987).

The effect of a long wave on growth of wind waves is quantified by investigating the ratio of the wind-wave growth rates at the frequency of the spectral peak both with and without long wave, namely $\gamma\left(\sigma_{p}\right)$ and $\gamma_{0}\left(\sigma_{0_{p}}\right)$ respectively. This ratio can be obtained 
using (4), and is

$$
\frac{\gamma\left(\sigma_{p}\right)}{\gamma_{0}\left(\sigma_{0_{p}}\right)}=\left(\frac{\sigma_{p}}{\sigma_{0_{p}}}\right)^{3} \frac{\tau_{t}}{\tau_{t o t}}
$$

which, from (16), becomes

$$
\frac{\gamma\left(\sigma_{p}\right)}{\gamma_{0}\left(\sigma_{0_{p}}\right)}=\left(\frac{\tau_{t}}{\tau_{\text {tot }}}\right)^{0.571} .
$$

Here we used the fact that $\beta$ varies only slowly with $c / U_{*}$ (see Fig. 2) and hence is nearly the same with and without the long wave.

Finally, equation (15) shows that the ratio of energy density of wind waves with and without long wave is

$$
\frac{E}{E_{0}}=\left(\frac{\tau_{t}}{\tau_{t o t}}\right)^{1.36}
$$

which is therefore strongly affected by the long wave.

At short fetch, the ratios of the peak frequency, growth rate and energy density of wind waves with and without long wave depend primarily on the long wave slope, $a_{L} k_{L}$, and the asymmetric pressure coefficient, $\alpha_{p}$. To make some initial rough estimates of the effect of long waves on these quantities, we approximate (13) to retain only the strongest dependency, namely

$$
\frac{\tau_{t}}{\tau_{\text {tot }}} \approx \frac{1}{1+\frac{1}{2}\left(a_{L} k_{L}\right)^{2} \alpha_{p}} .
$$

Variations in the ratios resulting from this approximation are plotted in Fig. 4 as a function of long wave slope for three different values of $\alpha_{p}$.

From these figures it is clear that addition of a long wave propagating in the wind direction causes a reduction in the energy density of wind waves, and that this effect becomes important as the long wave slope is increased. For a long wave slope of 0.1 , the energy density of wind waves is reduced by up to $40 \%$ of the value without long wave, but the growth rate at peak frequency is only reduced by up to $20 \%$ of the value without long wave. The strong effect of the reduction in the energy density of wind waves is from the exponent in (19), which is larger than the exponent in (18). The effect of long wave 
on the frequency of the spectral peak of wind waves is relatively weak for gentle long wave slopes as shown in Fig. 4a and reflected in (16). For instance, for a long wave slope, $a_{L} k_{L}=0.1$, the spectral peak frequency is increased by only $5 \%$. These results from the model agree qualitatively with laboratory measurements. Next the model is compared quantitatively with existing laboratory data.

\section{Comparison with laboratory experiments}

To compare the model results with laboratory data, we need to know the relevant values of four dimensionless parameters, namely $a_{L} k_{L}, \alpha_{p}, \sigma_{*}$ and $X_{*}-X_{*_{0}}$. None of the laboratory data provide values of $\alpha_{p}$ or of the growth rate coefficient, $\beta$. Given a large scatter of $\beta$ reported in the literature, it is actually a tricky question of how to choose an appropriate value of $\alpha_{p}$ or $\beta$. Our strategy is as follows. Given that the ratio $C_{L} / u_{*}$ for the laboratory experiments is in the range of 3 to 5 , we use a single value of $\alpha_{p}=80$ in the model. We then calculate the corresponding values of $\beta$ and $\epsilon_{L}$, and finally check if calculated $\beta$ values are in the range of data collated by Plant (1982) as shown in Fig. 2, namely $\beta=34 \pm 16$. Recall that we calculated $\beta$ from [see Eq. (4)]

$$
\beta=\frac{\alpha_{p}}{1+\frac{1}{2}\left(a_{L} k_{L}\right)^{2} \alpha_{p} \epsilon_{L}}
$$

Mitsuyasu and Honda (1982) measured systematically higher values of $\beta$ which suggests to us to use a relative higher value of $\alpha_{p}$. Indeed, a comparison of the model results with Mitsuyasu's (1966) experiment shows that a better agreement is obtained for $\alpha_{p}=160$, but again the responding values of $\beta$ are in the range reported by Mitsuyasu and Honda (1982). So $\alpha_{p}=80$ has been used in the comparisons with the results of Phillips and Banner (1974) and Donelan (1987). Both $\alpha_{p}=80$ and 160 have been used in the comparison with Mitsuyasu's (1966) data. 
a. Variation of the suppression of the wind waves with long wave slope

Phillips and Banner (1974) measured the total energy density, $E$, in wind waves at two fetches $(7.32 \mathrm{~m}$ and $10.36 \mathrm{~m})$ in the presence of a long wave, for different values of the long wave slope. They then obtained the ratio $E / E_{0}$, where $E_{0}$ is the energy density of the wind waves with no long wave. This ratio is calculated from (13) and (19), using the parameters of the experimental conditions, namely $\sigma_{L}=10.7 \mathrm{rad} \mathrm{s}^{-1}$ and $u_{*}=0.33$ $\mathrm{m} \mathrm{s}^{-1}$, corresponding to $\sigma_{*}=0.36, X_{*}-X_{*_{0}}=658.7$ (at the fetch of $7.32 \mathrm{~m}$ ) and $X_{*}-X_{*_{0}}=932.3$ (at the fetch of $10.36 \mathrm{~m}$ ). The value of the friction velocity of wind, $u_{*}$, was estimated from the measured mean-surface-wind drift $\left(\sim 0.18 \mathrm{~m} \mathrm{~s}^{-1}\right)$, which is about $0.55 u_{*}$ (Phillips and Banner 1974). As explained above, $\alpha_{p}$ is taken to be 80, from which $\beta$ lies in the range of $20\left(a_{L} k_{k}=0.2\right)$ to $50\left(a_{L} k_{L}=0.05\right)$ and 18 to 47 , respectively for the shorter and longer fetches. A comparison between the calculated values of $E / E_{0}$ with these parameters and the measured data is shown in Fig. 5. The solid and dashed lines represent, respectively, the calculated results for the shorter and longer fetches. The model results at both fetches are in fair agreement with the observations when the slope of the long wave is less than about 0.1. When the slope of the long wave is larger than about 0.1 , the model tends to under-estimate the reduction in the energy density of wind waves. However, the model shows the correct variation with both slope of the long wave and also fetch.

A comparison of the model with Mitsuyasu's experiment is shown in Fig. 6. The results of this experiment are summarized in Phillips and Banner (1974). To calculate $E / E_{0}$ from the model, (13) and (19), the following dimensionless parameters were used: $\sigma_{*}=0.35$ and $X_{*}-X_{*_{0}}=67.9$, which correspond to the experimental conditions of $u_{*} \sim 0.76 \mathrm{~m} \mathrm{~s}^{-1}, x-x_{0}=4 \mathrm{~m}$ and $\sigma_{L}=4.5 \mathrm{rad} \mathrm{s}^{-1}$. Again, the value of $u_{*}$ was obtained from the value of mean-surface-wind drift $\left(\sim 0.42 \mathrm{~m} \mathrm{~s}^{-1}\right)$ estimated by Mitsuyasu. The calculated ratios of $E / E_{0}$ are represented by the dashed line $\left(\alpha_{p}=80\right)$ and solid line 
$\left(\alpha_{p}=160\right)$ and the measured ratios are shown by the filled circles. The calculated values of $\beta$ are in the range $22\left(a_{L} k_{L}=0.20\right)$ to $40\left(a_{L} k_{L}=0.02\right)$ for $\alpha_{p}=80$, and 35 to 116 for $\alpha_{p}=160$. This figure shows that a better agreement with the observations is obtained for a larger value of $\alpha_{p}$. In particular, we note that weakly-nonlinear correction of the wave growth rate [see Eq. (4)] leads to the curve from the model decaying less rapidly with $a_{L} k_{L}$ for long wave slopes greater than about 0.1 , in agreement with the data. Hence we might regard these results as providing some support for the nonlinear analyses of wave growth given by van Duin (1996) and Belcher (1999).

The value of $\beta$ obtained in the model to produce Fig. 6 is high: up to 116 . This is a large value for the growth rate coefficient, for which we offer two possible explanations. Firstly, larger values of $\beta$ have been reported in laboratory experiments, particular those obtained in Mitsuyasu's wind-wave tank (see, for example, the data of Mitsuyasu and Honda (1982) replotted here in Fig. 2). Secondly, there may be a physical reason for the large values of $\beta$. If the Phillips-Banner mechanism does indeed enhance wave breaking at the long-wave crest, then this breaking leads to air-flow separation at the long-wave crest (Banner and Melville 1976), which in turn could lead to large effective value of $\beta$. Further work is needed to clarify this issue.

\section{b. Variation of the suppression of wind waves with fetch}

A more recent laboratory study of the effect of a long wave on the growth of wind waves has been undertaken by Donelan (1987). A comparison of the present model with his experimental results is particularly relevant since the experiment examined explicitly the sensitivity of the wind forcing of the short wind-waves to the presence of a long paddlegenerated wave. The experiment was performed in a wind-wave flume 100 meters long, 4.57 meters wide and 1.2 meters water deep. Long waves of frequency $0.527 \mathrm{~Hz}$ with initial slopes $a_{L} k_{L}=0$ (pure wind waves), 0.053 and 0.105 , were generated by a paddle. 
The water surface elevation was measured by nine capacitance wave staffs distributed along the $80-$ meter fetch. The total energy density $E$ of the wind waves for a particular long wave was fitted to a power law variation with fetch $x$, so that

$$
E=\alpha_{d} x^{\gamma_{d}}
$$

For instance, at a wind speed of $11 \mathrm{~m} \mathrm{~s}^{-1}$ (at 35 centimeters height), the values of $\alpha_{d}$ and $\gamma_{d}$ are, respectively, 0.015 and 1.56 (where $E$ is in $\mathrm{cm}^{2}$ and $x$ in $\mathrm{m}$ ). When a paddle wave of slope 0.105 was added to the wind waves, these values became 0.026 and 1.18. Consequently, when $a_{L} k_{L}=0.105$, the ratio $E / E_{0}$ decreases to $E / E_{0}=1.73 x^{-0.38}$, which is a function of fetch. Hence the energy density of the wind waves is reduced by a paddle wave of slope 0.105 by a factor of about 2.5 at a fetch of 50 meters.

On the other hand, the values of wind-wave growth-rate, estimated by Donelan from the pressure--surface elevation correlations in the cases of long waves plus wind waves were found to be similar to those measured in the case of wind waves only. This led Donelan to suggest that "the wind-input rate to the wind sea is rather insensitive to the presence of longer mechanically-generated waves of small steepness" - apparently at variance with the present model!

However, with the present model it is possible to explain Donelan's results. Indeed, when $a_{L} k_{L}=0.105$ and $\alpha_{p}=80$, the ratio of the growth rate of wind waves at the frequency of the peak in the wave spectrum can be estimated from (20) and (18) to give $\gamma / \gamma_{0} \approx 0.8$, and similarly $(20)$ and $(19)$ show that $E / E_{0} \approx 0.6$. Hence the reduction in $\gamma / \gamma_{0}$ is smaller than the reduction in $E / E_{0}$. At first this small reduction in $\gamma / \gamma_{0}$ is surprising, since the present model is predicated on the idea that the long wave reduces the turbulent stress near the water surface, which thence reduces wind generation of waves. The answer is that Donelan (1987) measured the growth rate of waves at the peak in the spectrum, and although the wave growth rate is affected by the long wave, so too is the frequency at the peak in the wave spectrum: see equations (16) and (17). 
More precise comparisons are presented in Fig. 7, where we plot the variation of $\gamma / \gamma_{0}$ and $E / E_{0}$ with dimensionless fetch. These ratios are calculated from (19) and (18), using in the model the parameters of Donelan's (1987) experimental conditions, namely $a_{L} k_{L}=0.105, \sigma_{L}=3.31 \mathrm{rad} \mathrm{s}^{-1}$ and $u_{*}=1 \mathrm{~m} \mathrm{~s}^{-1}$ with $\alpha_{p}=80$ as above (corresponding $\beta$ values varying from 34 at fetch $100 \mathrm{~m}$ to 50 at fetch $20 \mathrm{~m}$ ). Also shown is the powerlaw dependence $E / E_{0}=1.73 x^{-0.38}$ obtained empirically by Donelan (1987). The figure shows that according to the model the wind-wave growth rate is only slightly affected by the long waves, in agreement with the data given the difficulties in measuring $\gamma\left(\sigma_{p}\right)$. In addition the figure shows that the variation of $E / E_{0}$ with fetch obtained from the model agrees well with the laboratory data. We conclude that the present model captures the main trends in Donelan's (1987) data.

It is worthwhile comparing the results of Phillips and Banner (1974) with the same experimental data. They suggested that the long wave augments the wind-induced surface drift current near the crests, causing premature breaking of the short wind waves. Phillips and Banner estimate that the ratio, $r \approx\left(E / E_{0}\right)^{1 / 2}$, of the maximum wind wave amplitude to its maximum amplitude in the absence of the long waves is

$$
r=\frac{g}{g^{\prime}}\left(\frac{c_{c}-q_{\max }}{c_{0}-q_{0}}\right)^{2},
$$

where $g l$ is the effective acceleration of gravity at the crest of the long waves, $c_{0}$ and $C_{L}$ are the phase speed of the short waves (in the absence of the long waves) and the long waves, respectively, $c_{c}$ is the phase speed of the short waves at the crest of the long waves, $q_{0}$ is the surface drift in the absence of the long waves, and $q_{\max }$ is the augmented value of the wind drift. Equation (23) is an algebraic function of the three dimensionless parameters $c_{0} / C_{L}, u_{0} / C_{L}$ and $q_{0} / C_{L}$. These parameters were estimated as follows. Equation (14) was used to obtain $c_{0}=g / \sigma_{p}$ as a function of fetch, $x$, and friction velocity, $u_{*}$, namely

$$
c_{0}=0.3 u_{*}^{0.286} x^{0.357}
$$


Equation (24) was used to obtained $c_{0}$, and the measured long wave frequency was used to obtain $C_{L}$, thence we obtained $c_{0} / C_{L}$. The ratio $u_{0} / C_{L}$ was approximated here as $a_{L} k_{L}$. And finally, the surface drift was obtained from $q_{0} \approx 0.55 u_{*}$ as above. The energy density ratio $E / E_{0}$ (i.e., $\left.r^{2}\right)$ was then calculated from (23).

The results obtained from the Phillips-Banner model for $u_{*}=1 \mathrm{~m} \mathrm{~s}^{-1}$ the value in Donelan's experiment, and also for $u_{*}=0.5 \mathrm{~m} \mathrm{~s}^{-1}$, are plotted in Fig. 8. Also shown is the variation of $E / E_{0}$ with fetch obtained from the present model, and the values measured by Donelan (1987). It is clear from this figure that the Phillips-Banner model yields the wrong variation with fetch. In both cases $E / E_{0}$ from the Phillips-Banner model is increasing with fetch, whereas the laboratory data, and also the present model, show $E / E_{0}$ decreasing. Thus this data distinguishes the present model from the PhillipsBanner model.

\section{Implication for the effects of swell on wind waves on the ocean}

We have shown that the reduction in the energy density of wind waves caused by a long wave depends crucially on the long wave slope, $a_{L} k_{L}$, and the asymmetric pressure coefficient, $\alpha_{p}$, or the growth rate coefficient of the long wave, $\beta$. This latter parameter varies with the ratio $C_{L} / u_{*}$. In the laboratory experiments $C_{L} / u_{*}$ is small, typically less than 10, which, according to Fig. 2, leads to a large value of $\beta$ (typically $30-50$ ) and consequently a large value of $\alpha_{p}$, and hence, according to the present model, to a strong suppression of the short wind waves. However, in the field, ocean swell typically

has a large phase speed, $C_{L}$, and so the ratio of $C_{L} / u_{*}$ is usually larger than 25 . The corresponding value of $\beta$ is small and may even be negative (Fig. 2). Also the slopes of ocean swell are usually small, so Eq. (21) suggests $\alpha_{p} \approx \beta$ in the open ocean. Thus, the reduction in the wind wave energy caused by ocean swell is small. This is clearly illustrated in Fig. 9, where we plot the variations with swell slope of the ratio of energy 
densities of wind waves with and without swell for different values of $\alpha_{p}$. Clearly the reduction in the energy density of wind waves is small: less than $5 \%$ in the presence of swell with $\alpha_{p}=1$. This suggests that in the ocean the effects of swell on wind waves are very weak, certainly much weaker than the effect observed in the laboratory. Therefore, suppression of wind waves by ocean swell is extremely difficult to detect. This may explain why it has not yet been observed. Finally, we note that in any quantitative comparison of the present model with ocean observations, the fetch laws that have been used here, (14) and (15), which were obtained for laboratory data, would need to be replaced with fetch laws appropriate to the open ocean (see, for example, Komen et al. 1994, p 181).

\section{Conclusions}

We have developed a model for the suppression of short wind-generated waves by a train of long, mechanically-generated waves propagating in the wind direction. The model is based on the idea that the long wave exerts a drag on the air flow, which reduces the turbulent stress in the air flow that is available to generate wind waves. A crucial assumption of the model is that the only effect of the long wave is to reduce the turbulent wind stress, otherwise the fetch laws remain the same. Further work is needed to validate this assumption.

The model agrees reasonably well with existing laboratory measurements when an appropriate value is used for the asymmetric pressure coefficient of the long wave, $\alpha_{p}$, which is related to the growth rate coefficient, $\beta$. In the absence of values for either parameter for the laboratory experiments with which the model was compared, the choice of an appropriate value of $\alpha_{p}$ was based the strategy that the calculated $\beta$ values should be in the range 18 to 50, as suggested by data shown in Fig. 2. Indeed, in the comparisons with data we used a value of $\alpha_{p}=80$ (corresponding to a range of $\beta$ from 18 to 50), except in the comparison with Mitsuyasu (1966) where we also used a value of $\alpha_{p}=160$, 
corresponding to a range of $\beta$ from 35 to 116. Although these values of $\beta$ for $\alpha_{p}=160$ are large, as we have already argued, the very large value of $\alpha_{p}=160$ is consistent with values of $\beta$ measured by Mitsuyasu and Honda (1982), which are larger than other observations. Nevertheless the large value of $\alpha_{p}$ used here does require explanation. We suggest that the Phillips-Banner mechanism may be promoting breaking of the short wind waves on the crests of the long wave, which then leads to separation of the air flow at the crests of the long wave. Such separation is known to increase the wind-induced growth rate (see Belcher and Hunt 1998), and hence $\alpha_{p}$. Further research is needed to settle this issue.

Given that the value of $\beta$ exhibits a relatively large scatter, as illustrated in Fig. 2, which reflects the difficulty in measuring this parameter, it is useful to make further comments on the sensitivity of the model results on the value of $\alpha_{p}$. From the present model it becomes clear that the larger the value of $\alpha_{p}$ or $\beta$ of the long wave is, the stronger the suppression of the wind waves would be. The actual range of the model results will be dependent upon other three dimensionless parameters, namely $a_{L} k_{L}, \sigma_{*}$ and $X_{*}-X_{*_{0}}$. However, the general trends of the model results are in line with available laboratory data. For instance, according to the model, for the two extreme values of $\beta$, namely 18 and 50, the ratio of the total energy density of wind waves with and without the long wave, $E / E_{0}$, takes values of 0.65 (when $\beta=50$ ) to 0.87 (when $\beta=18$ ) for Donelan's (1987) data. Hence the suppression of the wind waves by the long wave is fairly well predicted by the model. With the choices of $\alpha_{p}$ used in the paper the values of $\beta$ obtained from the model always lie within the range $34 \pm 16$ suggested by Plant (1982) except in the comparison with Mitsuyasu (1966) for which a larger value is used for the reasons described above. Nevertheless it must be emphasized that for any application of the model a caution should be taken as for whether an appropriate value of $\alpha_{p}$ or $\beta$ is used.

Notwithstanding this shortcoming, the model has strengths over previous models 
suggested to explain this phenomenon. Firstly, the model gives roughly the correct variation of $E / E_{0}$, the ratio of the energy density of the wind waves with and without the long wave, with fetch when compared with the data of Donelan (1987). In addition, the model explains why the rate of growth of waves at the peak in the wind-wave spectrum is almost unaffected by the long wave. When Donelan (1987) found this result in his data, he concluded that the suppression of the wind waves could not be due to coupling with the wind. The work presented here, which is based on the idea that it is the coupling between the wind and long wave that suppresses the wind waves, shows that both the growth rate of the waves at the peak in the spectrum and also the frequency of the waves at the peak in the spectrum are affected, and that these two effects nearly cancel oneanother. Finally, and perhaps most significantly, the present model explains why swell on the ocean, which travels at a phase speed that is close to the wind speed and hence has a small value of $\alpha_{p}$, leaves the wind sea almost unaffected. Hence the suppression of wind waves observed so strongly in the laboratory does not occur in the ocean.

The success of the present model lends support to the idea that long wavelength waves absorb momentum from the wind leaving the short wavelength waves exposed to a smaller turbulent wind stress, and hence perhaps lends indirect support for the idea that a similar process occurs within a spectrum of waves, as suggested by Makin et al. (1995) in their calculation of the drag of the sea surface due to a spectrum of waves.

Acknowledgements. We are grateful to Vladimir Makin for useful conversations during the course of this work, and also to Fabien Remy and Jean-Paul Giovanangeli for kindly providing us with their experimental data shown in Fig. 3. We would also like to thank one of the referees for pointing out an error in an early version of the manuscript. This work was supported by the EC Environment Programme under the ASPEN project, contract ENV4-CT97-0460. 


\section{References}

Banner, M. L. and W. K. Melville, 1976: On the separation of air flow over water waves. J. Fluid Mech., 77, 825-842.

Belcher, S. E., 1999: Wave growth by non-separated sheltering. Euro. J. Mech. /B Fluids, 18, 447-462.

Belcher, S. E. and G. Chen 1999: Effects of swell on wind-wave generation. Paper presented at Air-Sea Interface Symposium, Sydney, January 11-15, 1999.

Belcher, S. E. and J. C. R. Hunt, 1993: Turbulent shear flow over slowly moving waves. J. Fluid Mech., 251, 109-148.

Belcher, S. E. and J. C. R. Hunt, 1998: Turbulent flow over hills and waves. Ann. Rev. Fluid Mech., 30, 507-538.

Donelan, M. A., 1987: The effect of swell on the growth of wind waves. Johns Hopkins APL Tech. Dig., 8, 18-23.

Duin, C. A. van, 1996: An asymptotic theory for the generation of nonlinear surface gravity waves by turbulent air flow. J. Fluid Mech., 320, 287-304.

Komen, G. J., L. Cavaleri, M. A. Donelan, K. Hasselmann, S. Hasselmann and P. A. E. M. Janssen, 1994: Dynamics and Modelling of Ocean Waves. Cambridge University Press, $532 \mathrm{pp}$.

Makin, V. K. and V. M. Kudryavtsev, 1999: Coupled sea surface-atmosphere model. Part 1. Wind over waves coupling. J. Geophys. Res., 104(C4), 7613-7624.

Makin, V. K., V. M. Kudryavtsev and C. Mastenbroek, 1995: Drag of the sea surface. Boundary-Layer Meteorol., 73, 159-182.

Masson, D., 1993: On the nonlinear coupling between swell and wind waves. J. Phys. Oceanogr., 23, 1249-1258.

Mastenbroek, C., 1996: Wind-wave interaction. Ph.D. thesis, Delft Technical University, 119 pp. 
Mitsuyasu, H., 1966: Interactions between water waves and wind (1). Rep. Inst. Appl. Mech. Kyushu Univ., 14, 67-88.

Mitsuyasu, H. and T. Honda, 1982: Wind-induced growth of water waves. J. Fluid Mech., 123, 425-442.

Mitsuyasu, H. and K. Rikiishi, 1978: The growth of during-limited wind waves. J. Fluid Mech., 85, 705-730.

Phillips, O. M., 1977: The Dynamics of the Upper Ocean. 2nd Ed., Cambridge University Press, $336 \mathrm{pp}$.

Phillips, O. M. and M. L. Banner, 1974: Wave breaking in the presence of wind drift and swell. J. Fluid Mech., 66, 625-640.

Plant, W. J., 1982: A relationship between wind stress and wave slope. J. Geophys. Res., 87, 1961-1967.

Remy, F. and J. P. Giovanangeli, 1999: Wind stress over simulated complex sea states. Paper presented at Air-Sea Interface Symposium, Sydney, January 11-15, 1999.

Townsend, A. A., 1972: Flow in a deep turbulent boundary layer disturbed by water waves. J. Fluid Mech., 55, 719-735.

Wright, J. W., 1976: The wind drift and wave breaking. J. Phys. Oceanogr., 6, 402-405. 


\section{List of Figures}

1 Schematic of (a) the configuration with wind blowing over wind waves and paddle waves, and (b) the vertical profiles of contributions to the total stress, $\tau_{t o t} \ldots \ldots \ldots \ldots \ldots \ldots \ldots \ldots \ldots \ldots \ldots \ldots \ldots \ldots \ldots \ldots \ldots \ldots$

2 Variation of wave growth-rate coefficient, $\beta$, with $c / u_{*}$. Filled triangles: linear theory for slow and fast waves (Belcher 1999); joined open circles: nonlinear numerical computation with second-order closure (Mastenbroek 1996); filled circles: laboratory measurements of wind-ruffled paddle-generated waves (Mitsuyasu and Honda 1982); other symbols: data collated by Plant (1982). . . . . . . . . . . . . . . .

3 Total stress, $\tau_{\text {tot }} / \rho_{a}\left(\right.$ in $\left.\mathrm{m}^{2} \mathrm{~s}^{-2}\right)$, measured 24 centimeters above the water surface, for different slopes of long, oblique waves (Remy and Giovanangeli 1999)

4 The calculated variation in the ratios of (a) peak frequency; (b) growth rate at the spectral peak frequency; (c) energy density of wind waves with and without long wave as a function of long wave slope, $a_{L} k_{L}$, for three different values of $\alpha_{p}$ ( $\alpha_{p}=20$ : dash-dotted line; 40: dashed line; 100: solid line). . . . . . . . . . . . . . . . . .

5 A comparison of the ratio $E / E_{0}$ predicted by the model with that measured by Phillips and Banner (1974). The calculated ratios are represented by lines, solid line: at a fetch of $7.32 \mathrm{~m}$; dashed line: at a fetch of $10.36 \mathrm{~m}$. The measured values are shown by symbols, upward pointing triangles: at a fetch of $7.32 \mathrm{~m}$; squares: at a fetch of $10.36 \mathrm{~m} . \ldots . . . . . . .31$ 
6 A comparison of the calculated ratio $E / E_{0}$ (dashed line: $\alpha_{p}=80$; solid line: $\alpha_{p}=160$ ) with the measured ratio (filled circles) by Mitsuyasu (1966). 32

7 A comparison of the calculated ratio $E / E_{0}$ (solid line) as a function of dimensionless fetch with the measured ratio (dashed line) by Donelan (1987). The calculated ratio of the growth rate of wind waves (at the spectral peak frequency) with and without long wave, versus dimensionless fetch, is represented by the dash-dotted line. . . . . . . . . . . . .

8 A comparison of the present model with the theory of Phillips and Banner (1974). The calculated ratio $E / E_{0}$ as a function of dimensionless fetch by the present model is shown by the solid line (as in Fig. 7). The ratios $E / E_{0}$ predicted by the theory of Phillips and Banner for two different values of wind friction velocity, $u_{*}$, are represented by the dash-dotted line $\left(u_{*}=0.5\right.$ $\left.\mathrm{m} \mathrm{s}^{-1}\right)$ and the long-dashed line $\left(u_{*}=1 \mathrm{~m} \mathrm{~s}^{-1}\right)$. The measured ratio $E / E_{0}$ by Donelan (1987) is shown by the dashed line (as in Fig. 7) . . . . . . . . 34

9 The ratio of energy density of wind waves, $E / E_{0}$, versus long wave slope, $a_{L} k_{L}$, for different values of the growth coefficient, $\alpha_{p}$, as indicated on the

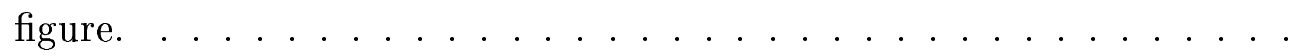




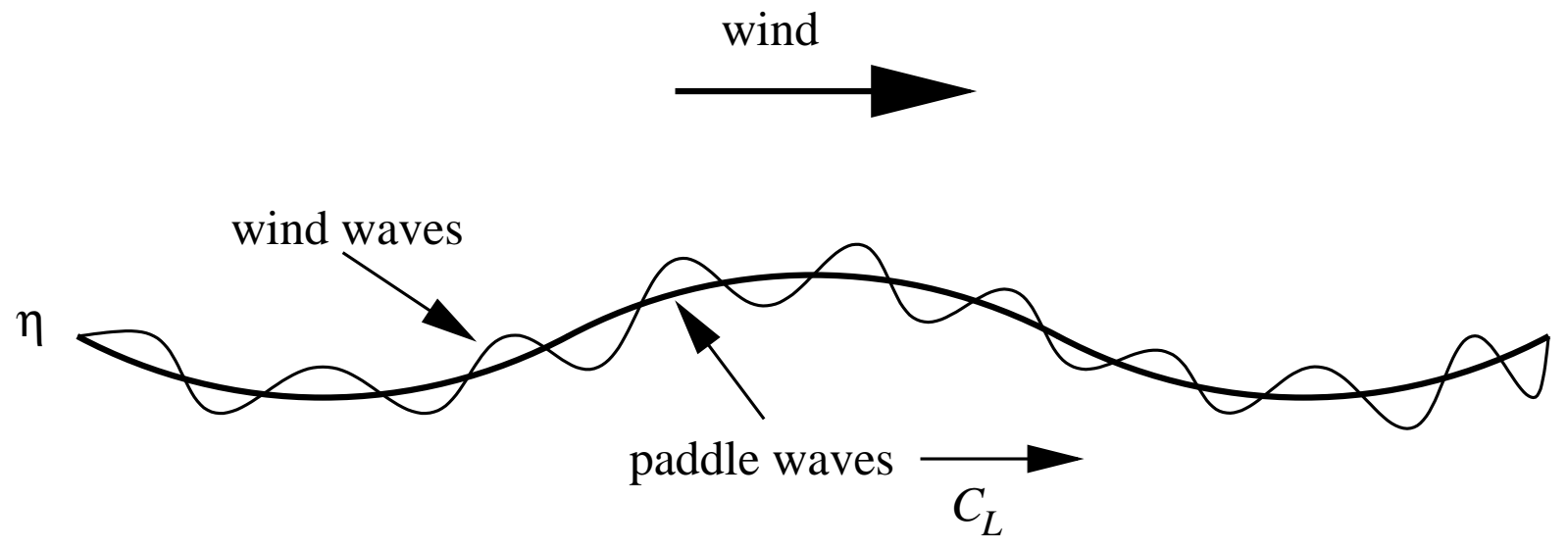

(a)

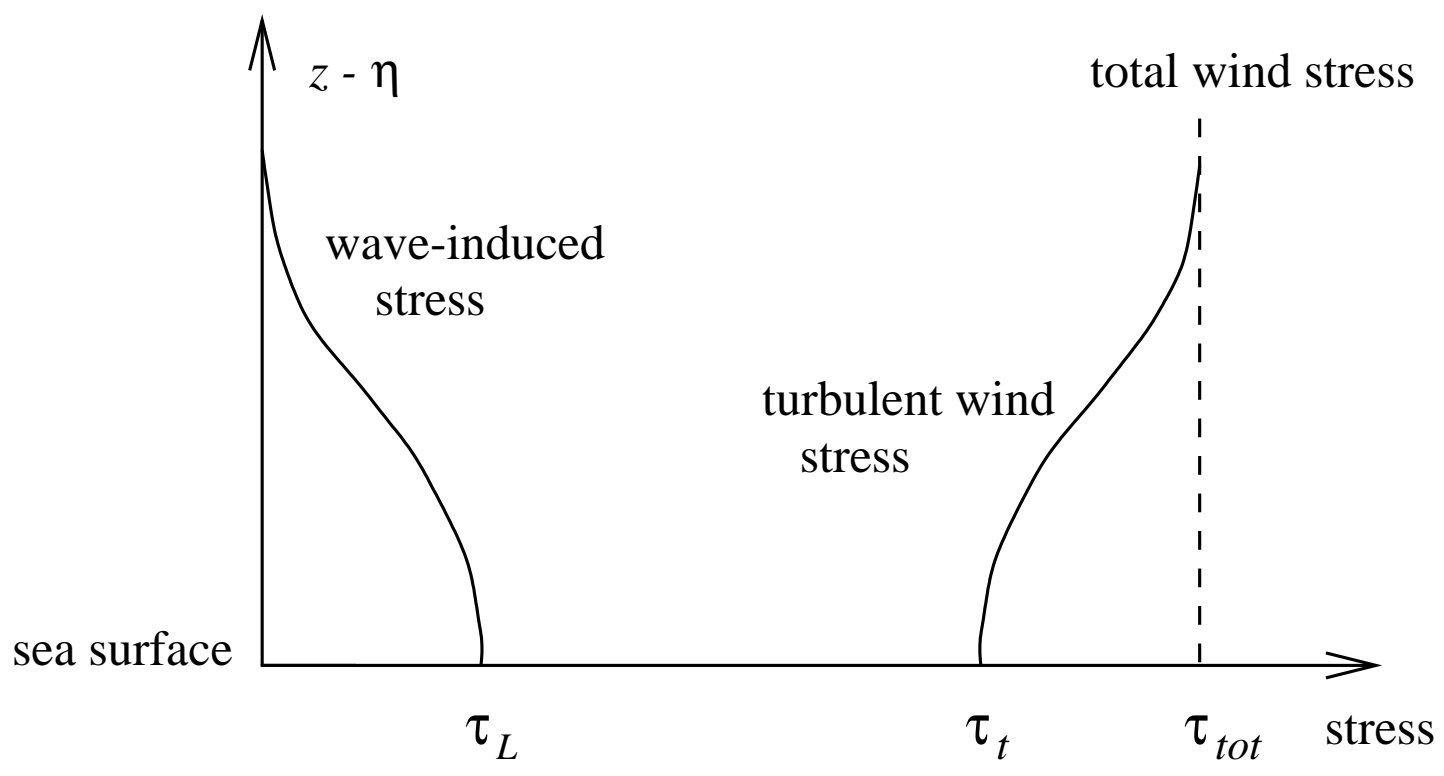

(b)

Figure 1: Schematic of (a) the configuration with wind blowing over wind waves and paddle waves, and (b) the vertical profiles of contributions to the total stress, $\tau_{\text {tot }}$. 


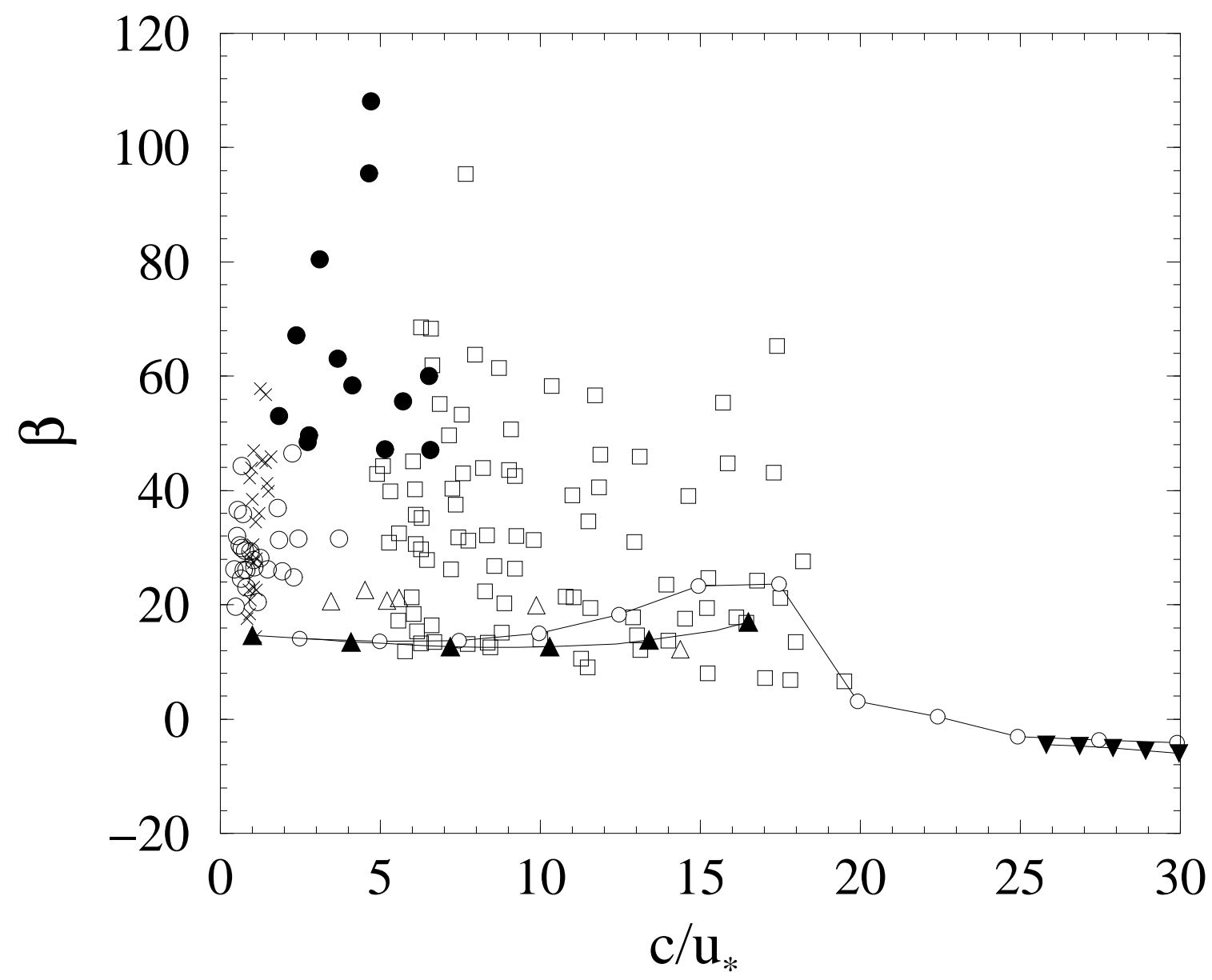

Figure 2: Variation of wave growth-rate coefficient, $\beta$, with $c / u_{*}$. Filled triangles: linear theory for slow and fast waves (Belcher 1999); joined open circles: nonlinear numerical computation with second-order closure (Mastenbroek 1996); filled circles: laboratory measurements of wind-ruffled paddle-generated waves (Mitsuyasu and Honda 1982); other symbols: data collated by Plant (1982). 


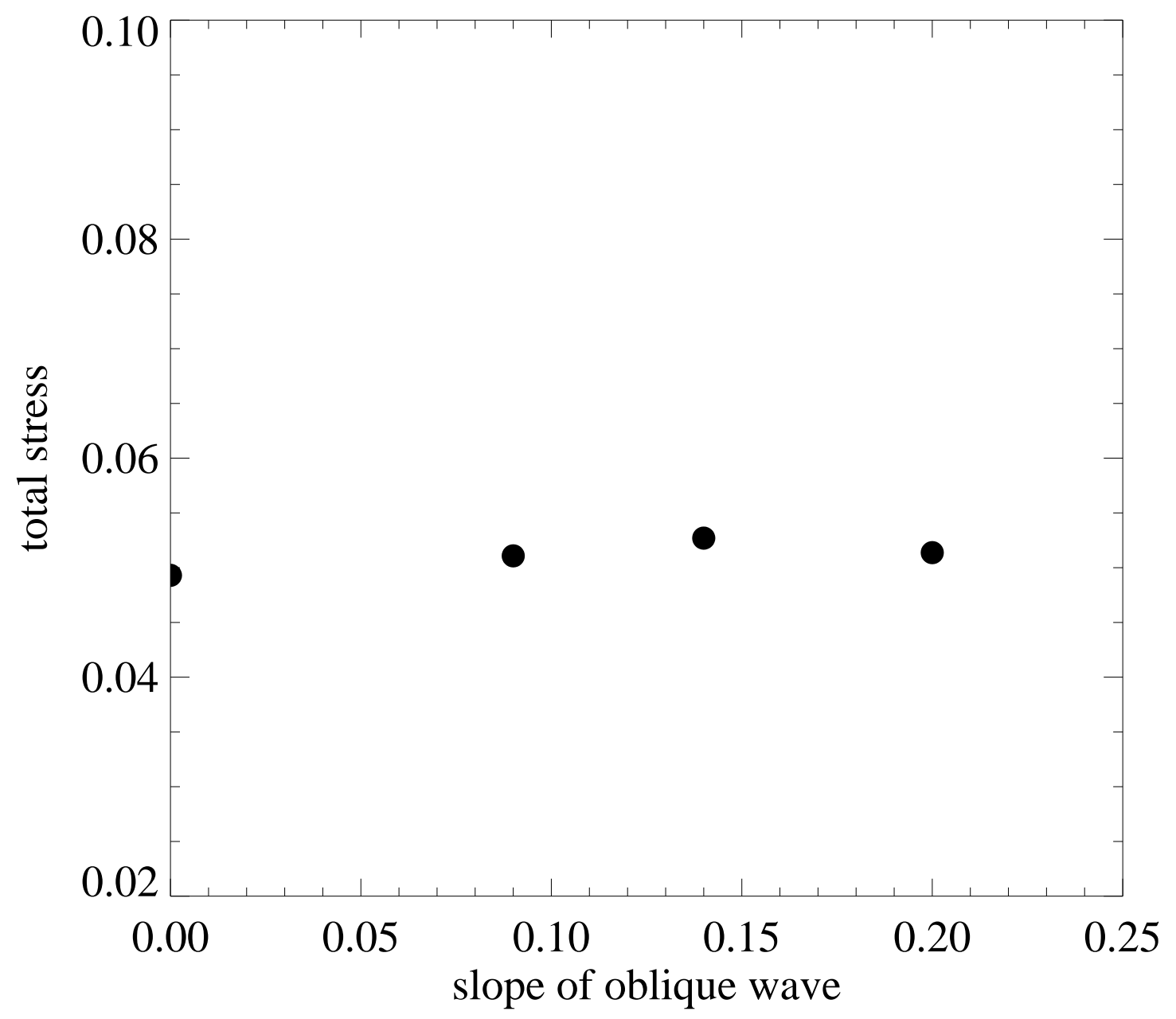

Figure 3: Total stress, $\tau_{\text {tot }} / \rho_{a}$ (in $\mathrm{m}^{2} \mathrm{~s}^{-2}$ ), measured 24 centimeters above the water surface, for different slopes of long, oblique waves (Remy and Giovanangeli 1999). 


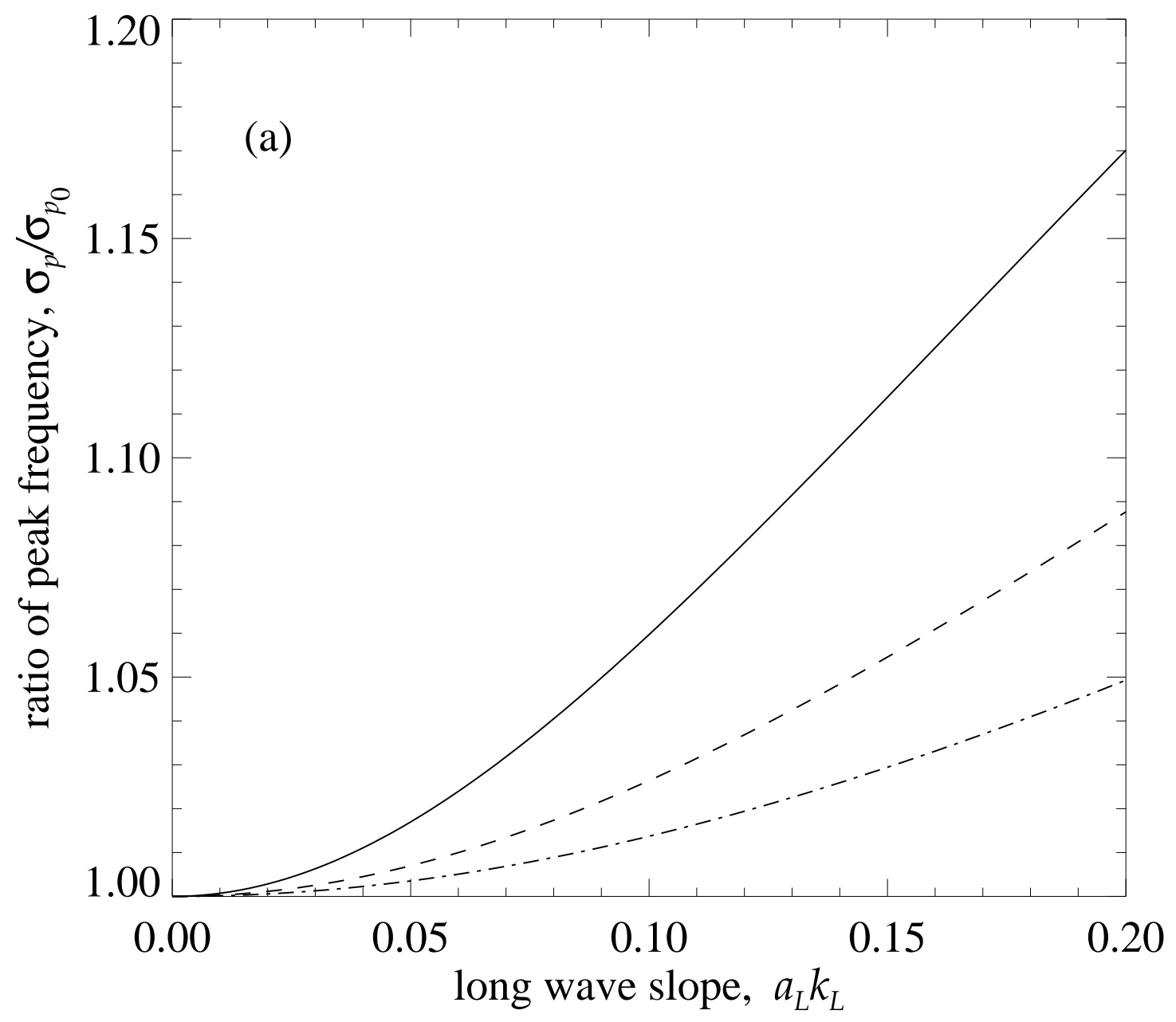

Figure 4a 


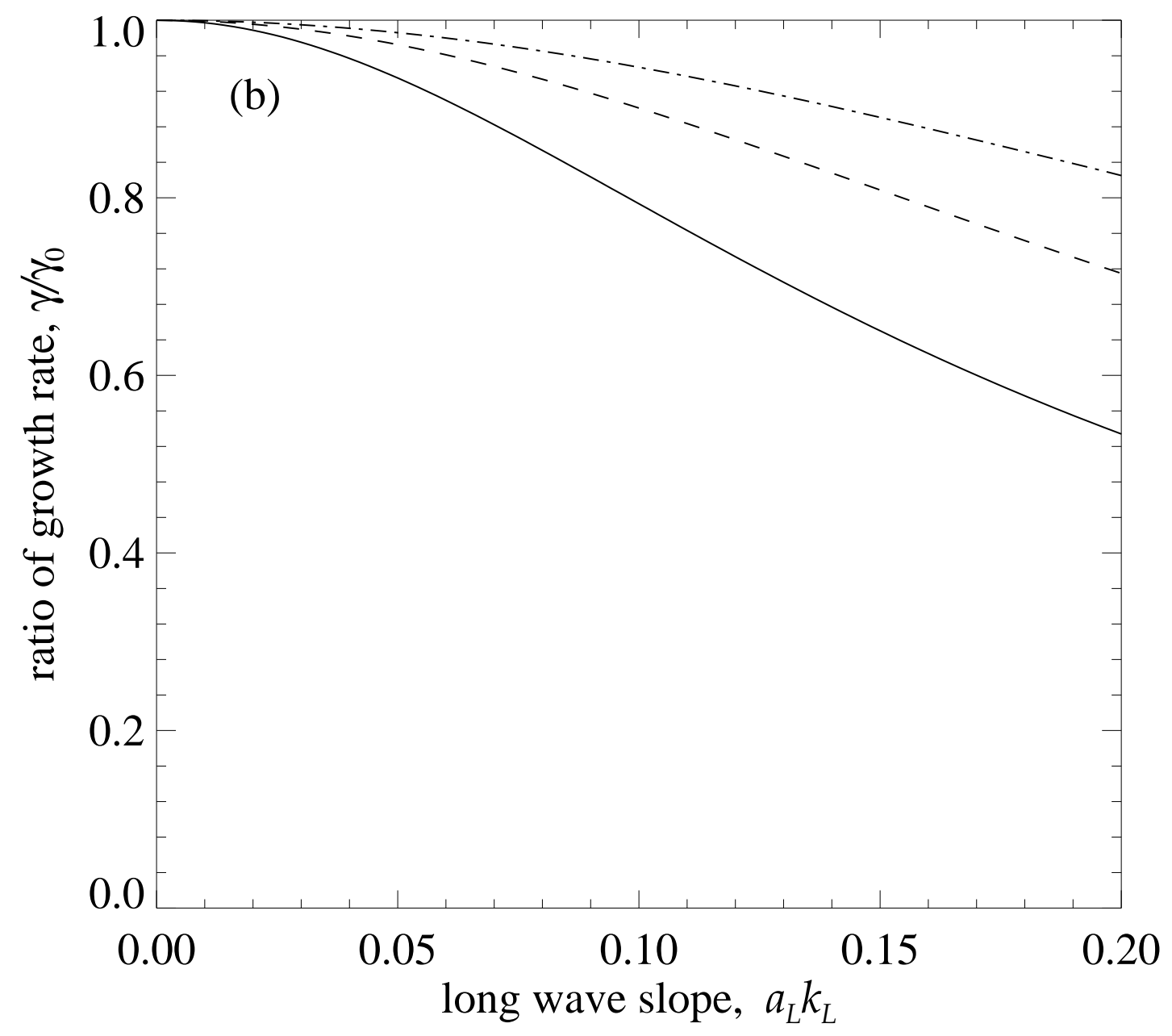

Figure 4b 


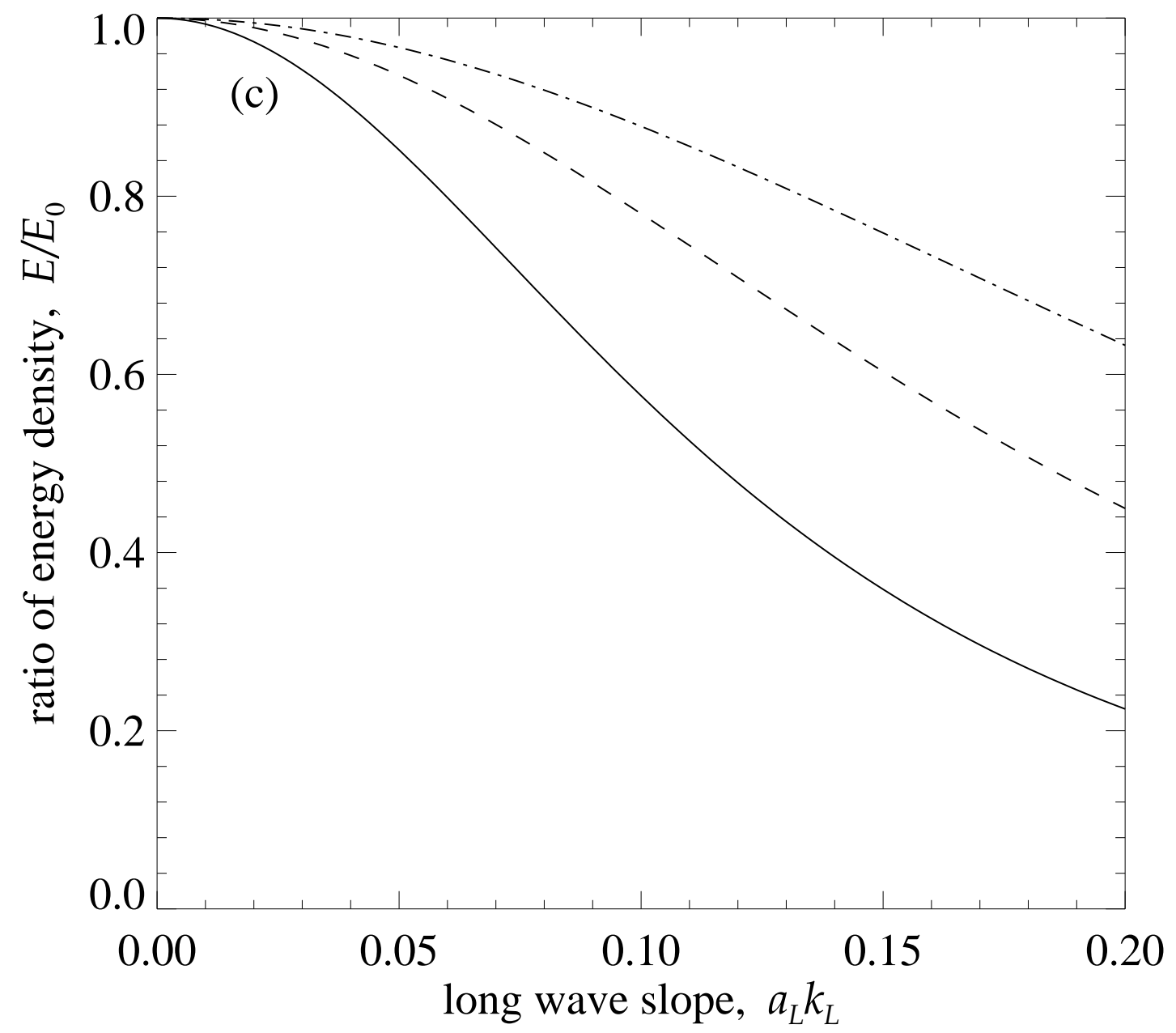

Figure 4: The calculated variation in the ratios of (a) peak frequency; (b) growth rate at the spectral peak frequency; (c) energy density of wind waves with and without long wave as a function of long wave slope, $a_{L} k_{L}$, for three different values of $\alpha_{p}\left(\alpha_{p}=20\right.$ : dash-dotted line; 40: dashed line; 100: solid line). 


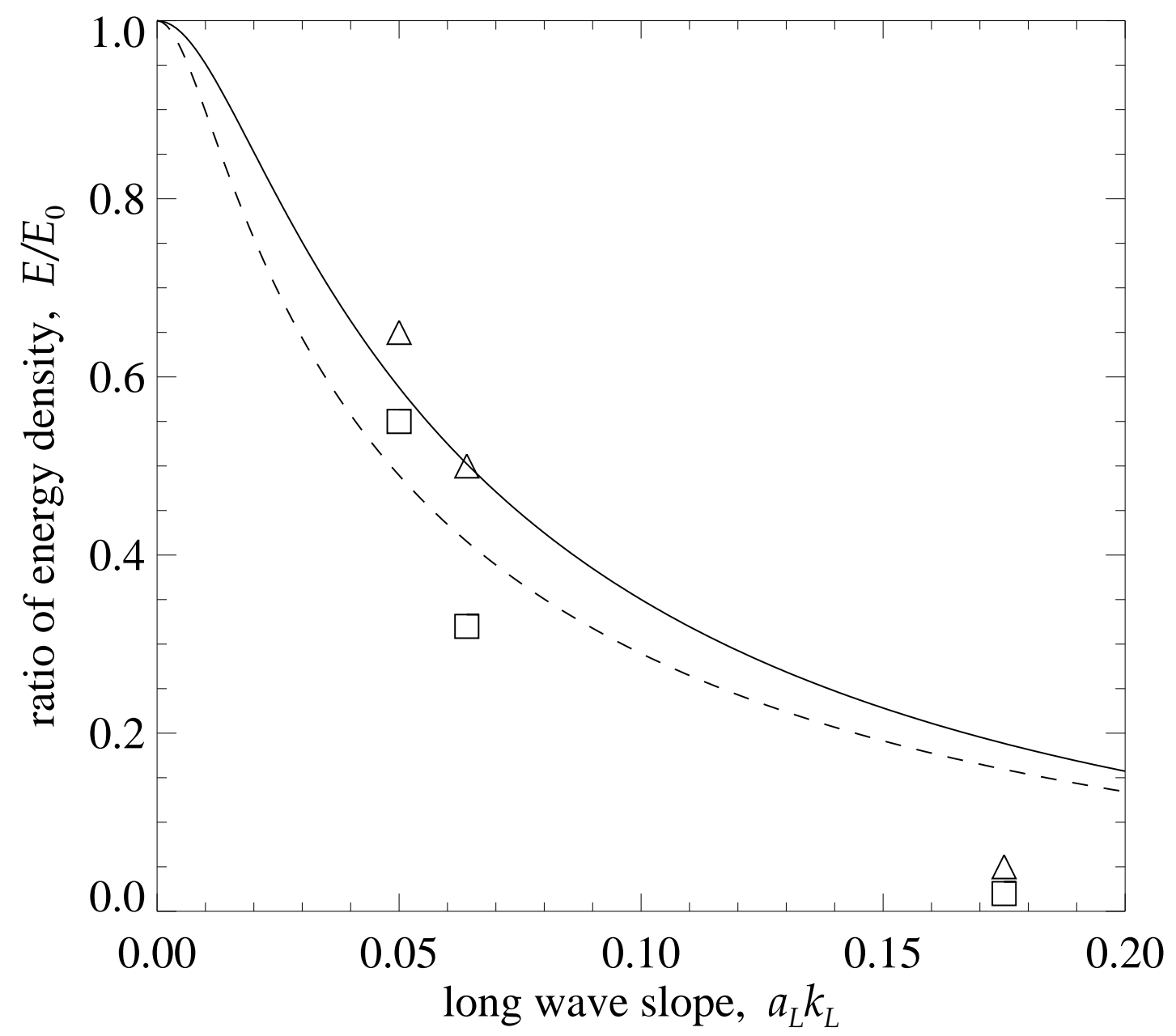

Figure 5: A comparison of the ratio $E / E_{0}$ predicted by the model with that measured by Phillips and Banner (1974). The calculated ratios are represented by lines, solid line: at a fetch of $7.32 \mathrm{~m}$; dashed line: at a fetch of $10.36 \mathrm{~m}$. The measured values are shown by symbols, upward pointing triangles: at a fetch of $7.32 \mathrm{~m}$; squares: at a fetch of $10.36 \mathrm{~m}$. 


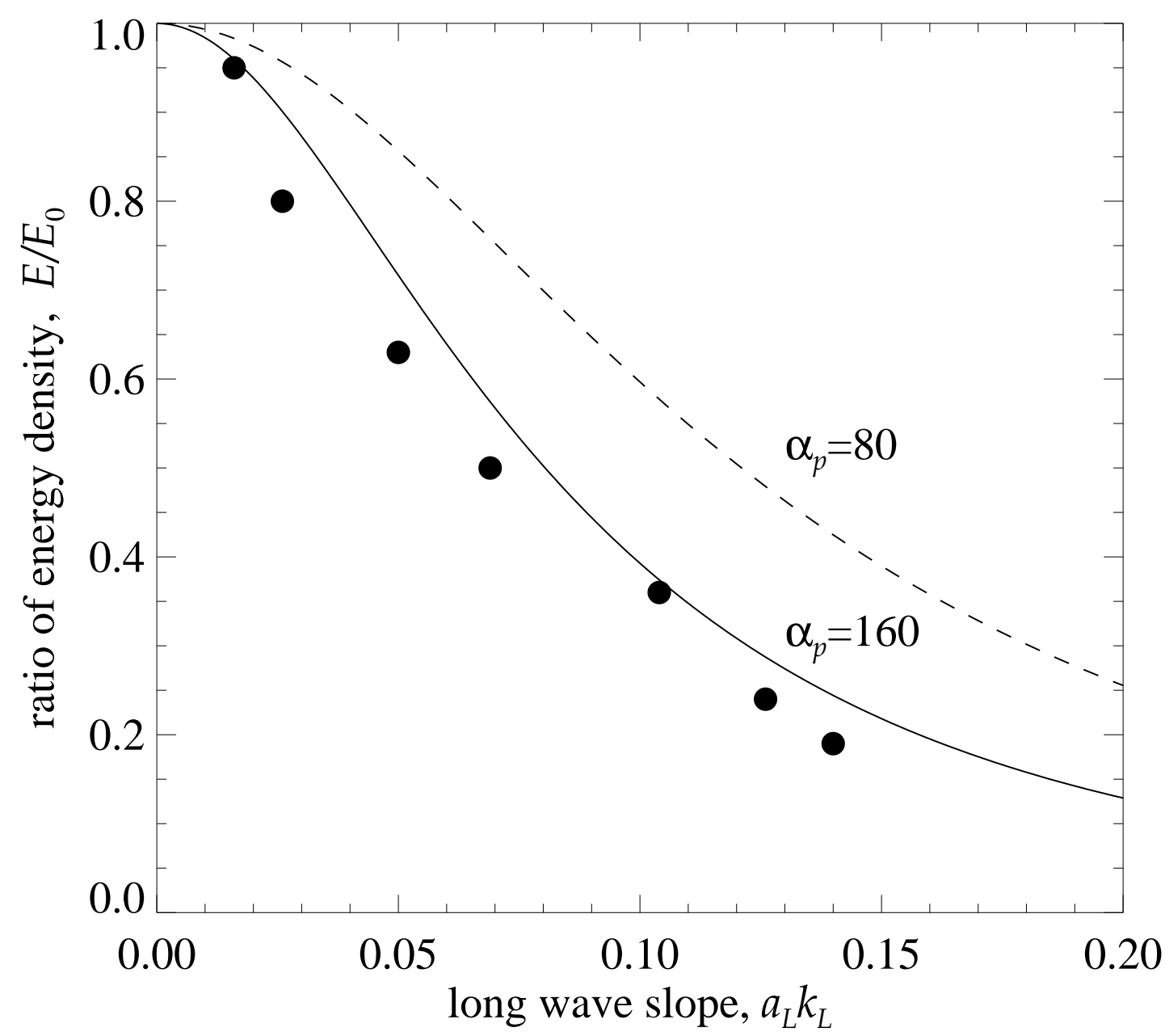

Figure 6: A comparison of the calculated ratio $E / E_{0}$ (dashed line: $\alpha_{p}=80$; solid line: $\alpha_{p}=160$ ) with the measured ratio (filled circles) by Mitsuyasu (1966). 


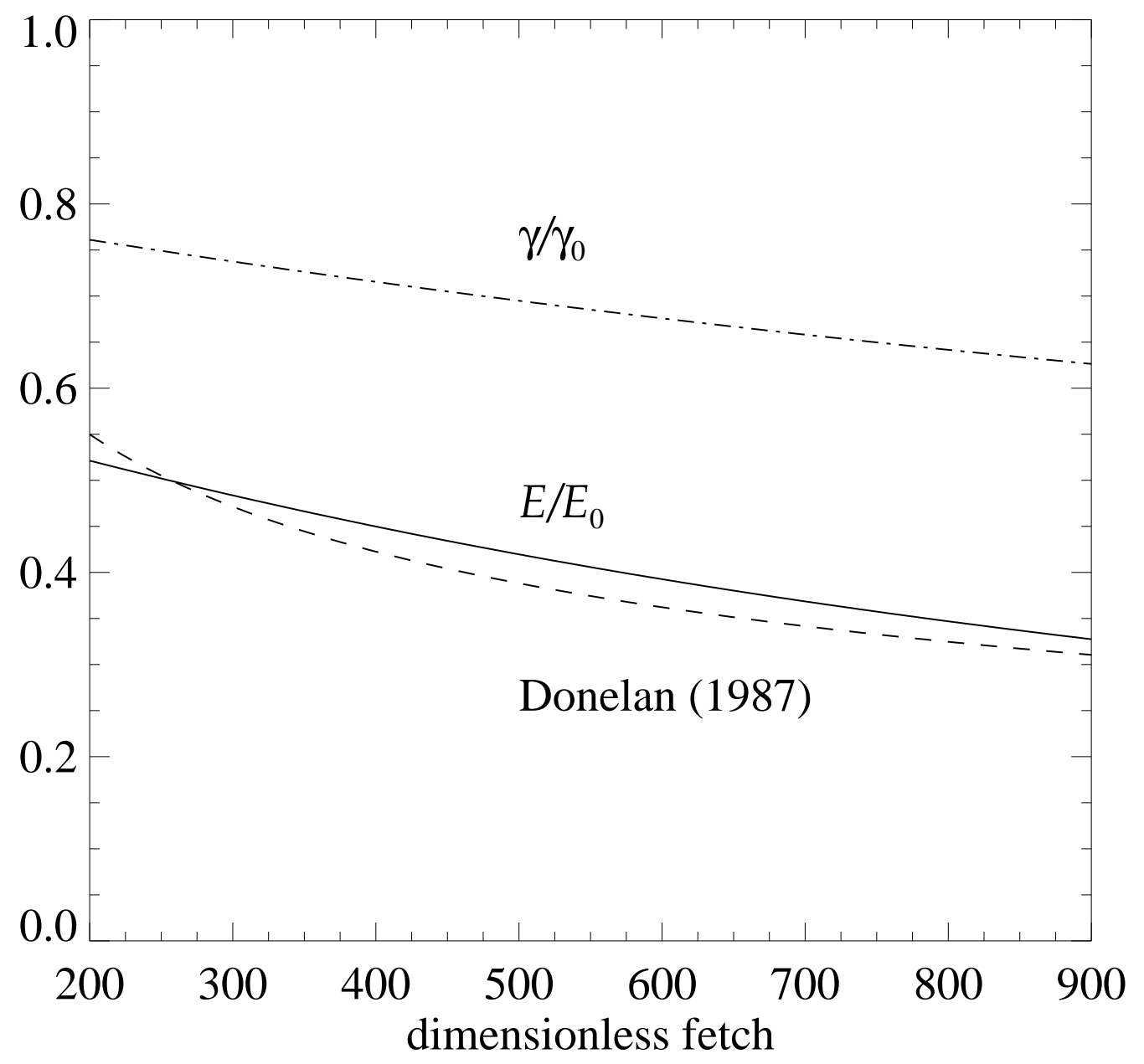

Figure 7: A comparison of the calculated ratio $E / E_{0}$ (solid line) as a function of dimensionless fetch with the measured ratio (dashed line) by Donelan (1987). The calculated ratio of the growth rate of wind waves (at the spectral peak frequency) with and without long wave, versus dimensionless fetch, is represented by the dash-dotted line. 


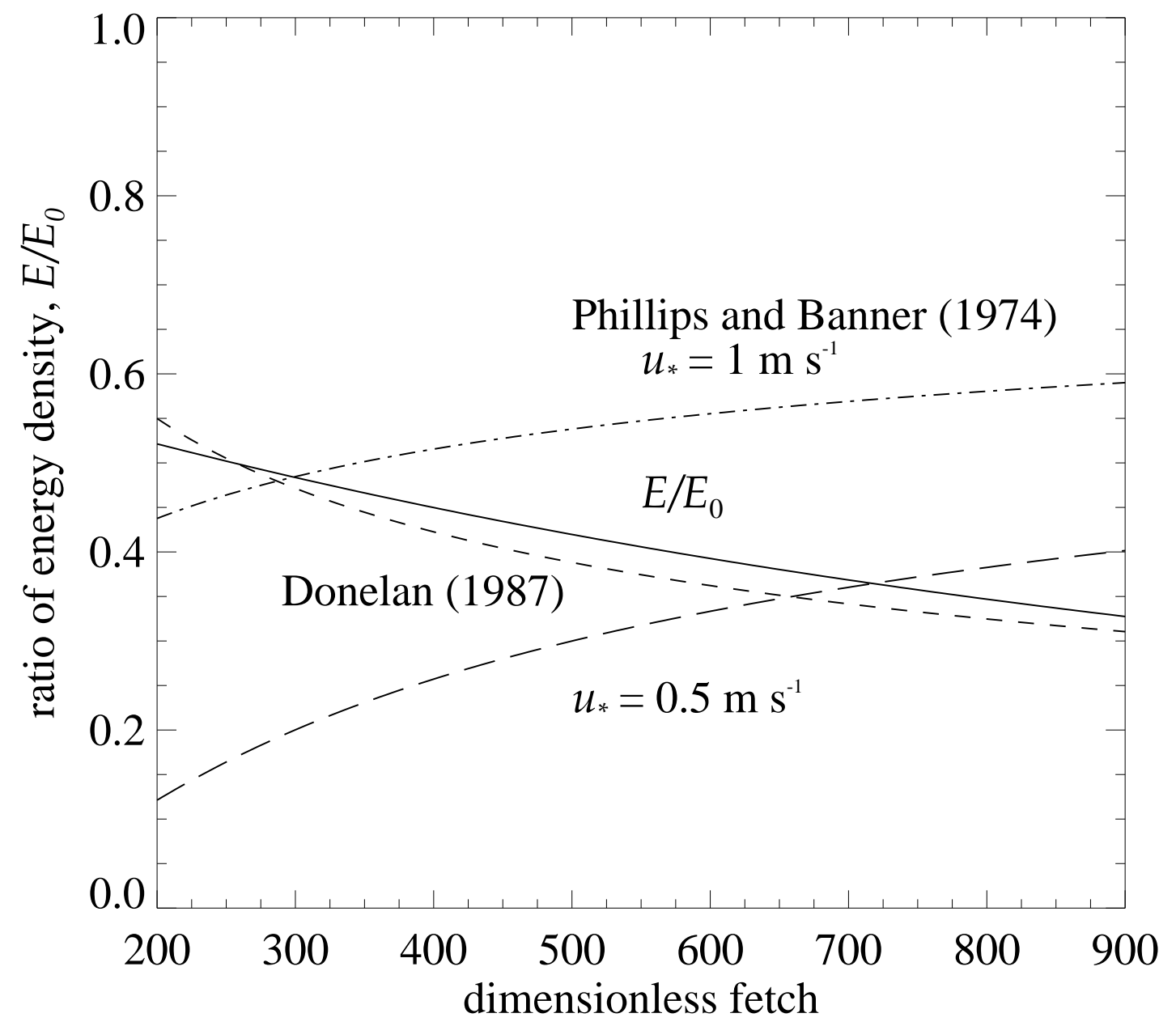

Figure 8: A comparison of the present model with the theory of Phillips and Banner (1974). The calculated ratio $E / E_{0}$ as a function of dimensionless fetch by the present model is shown by the solid line (as in Fig. 7). The ratios $E / E_{0}$ predicted by the theory of Phillips and Banner for two different values of wind friction velocity, $u_{*}$, are represented by the dash-dotted line $\left(u_{*}=0.5 \mathrm{~m} \mathrm{~s}^{-1}\right)$ and the long-dashed line $\left(u_{*}=1 \mathrm{~m} \mathrm{~s}^{-1}\right)$. The measured ratio $E / E_{0}$ by Donelan (1987) is shown by the dashed line (as in Fig. 7). 


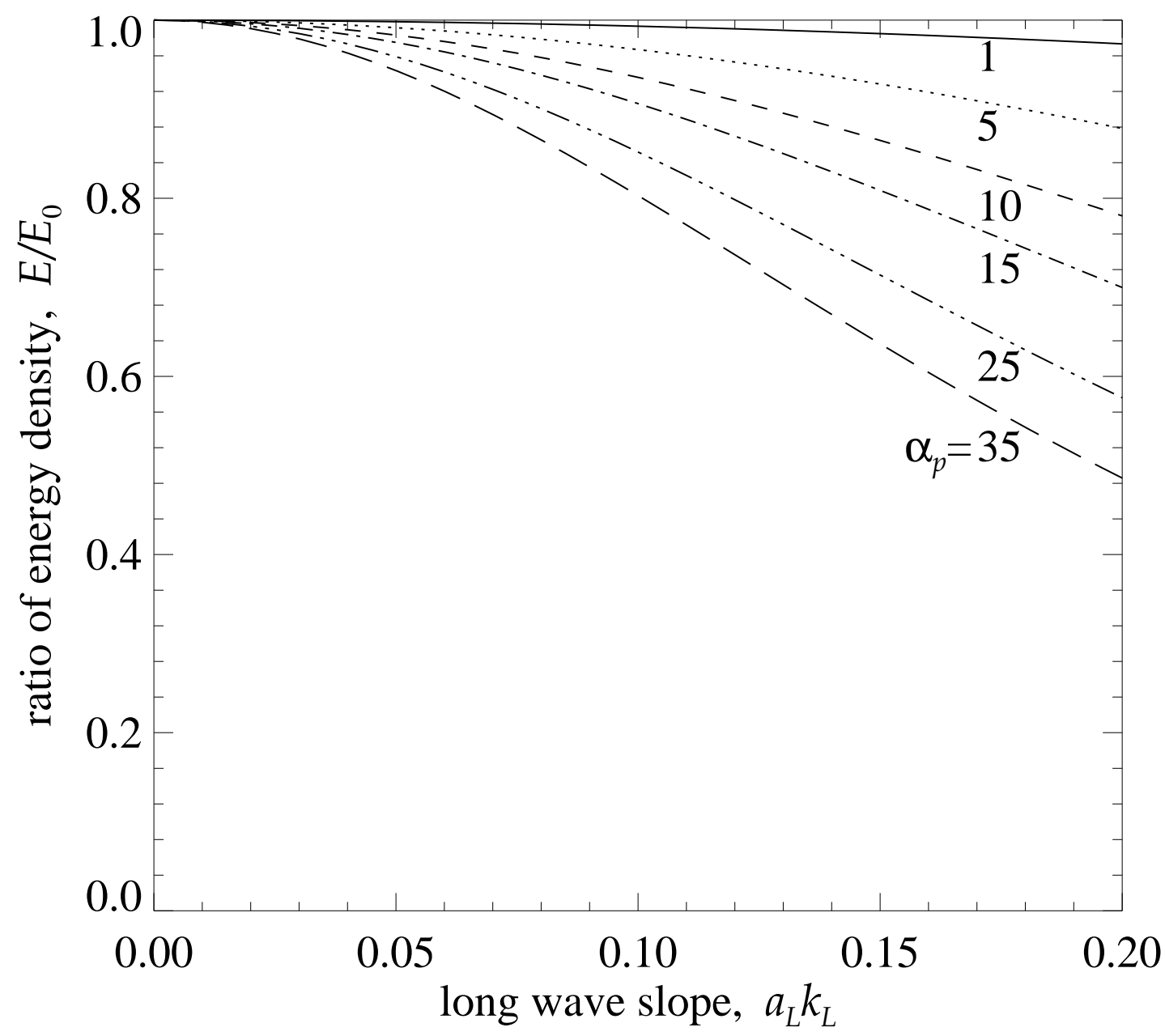

Figure 9: The ratio of energy density of wind waves, $E / E_{0}$, versus long wave slope, $a_{L} k_{L}$, for different values of the growth coefficient, $\alpha_{p}$, as indicated on the figure. 